\title{
Effects of Rician Faded and Log-Normal Shadowed Signals on Spectrum Efficiency in Microcellular Radio
}

\author{
Ramjee Prasad, Senior Member, IEEE, and Adriaan Kegel, Member, IEEE
}

\begin{abstract}
An assessment of spectrum efficiency for a microcellular land mobile radio system is presented by considering the desired signal as (fast) Rician fading with (slow) log-normal shadowing and co-channel interfering signals as uncorrelated (fast) Rayleigh fading superimposed over (slow) log-normal shadowing. Spectrum efficiency is defined in terms of reuse distance, i.e., cluster size, traffic intensity, bandwidth of the system, and area of a cell by considering co-channel interference probability. The expression for co-channel interference probability is derived using appropriate path-loss law for microcells for four different cases, viz.: i) Rician plus log-normal desired signal and Rayleigh plus log-normal interfering signals; ii) Rician desired signal and Rayleigh fading plus log-normal shadowing interfering signals; iii) Rician desired signal and Rayleigh interfering signals; and iv) both desired and interfering signals as Rician fading. Finally, the performance of a microcellular system is compared with that of a conventional macrocellular system.
\end{abstract}

\section{INTRODUCTION}

$\mathbf{M}$ OBILE radio communications are already used in practice by several million people worldwide, with a very high rate of annual increment. This fast-growing number of users demands efficient use of the scarce frequency spectrum. In order to overcome this problem, cellular radio systems are being developed [1], [2]. The cellular systems are classified into three categories, depending on the size of the cells, as follows: i) macrocells of 2 to $20 \mathrm{~km}$ diameter with antennas radiating power in the range of 0.6 to $10 \mathrm{~W}$ from the top of tall buildings; ii) microcells of 0.4 to $2 \mathrm{~km}$ diameter with antennas at street-lamp elevation and radiating power less than $20 \mathrm{~mW}$; and iii) picocells of much smaller size (20 to $400 \mathrm{~m}$ diameter) especially suited for indoor radio communications (e.g. offices, research laboratories, hospitals, modern factories, University campuses, etc.) with antennas placed on top of a bookshelf and radiating power on the order of a few $\mathrm{mW}$.

The performance analysis of macrocellular systems is reported in numerous research papers, e.g. [3]-[5]. Since microcells provide enhanced spectrum efficiency and low power consumption of handhelds, recently researchers have turned their attention to investigate outage probability [6] and spectrum efficiency of microcellular radio [7]-[9]. In

Manuscript received January 27, 1992; revised March 18, 1992, and August 28,1992 . This is an expanded version of work originally presented at the 41 st IEEE Vehicular Technology Conference, St. Louis, MO, May 1991, and the 42nd IEEE Vehicular Technology Conference, Denver, CO, May 1992.

The authors are with the Telecommunications and Traffic Control Systems Group, Delft University of Technology, 2600 GA Delft, The Netherlands.

IEEE Log Number 9208472.
[6] the outage probability is evaluated, assuming the desired signal as Rician fading, and assuming interfering signals as Rayleigh distributed without considering the path-loss law. The spectrum efficiency is calculated in [7], assuming both desired and interfering signals as Rician faded and fourth path power law. The authors have presented the spectrum efficiency analysis of a microcellular radio channel [8], [9] by considering the turning point in path-loss slope with the desired and interfering signals as Rician and Rayleigh distributed, respectively. Propagation measurements characterize the microcellular environment such that the signal fading can be decomposed into a path-loss component together with a slow varying log-normal shadowed component and a fast varying Rician component [10]-[15].

This paper presents a mathematical model to analyze the spectrum efficiency, assuming the desired signal within a cell as Rician faded with log-normal shadowed and interfering signals from co-channel cells as Rayleigh faded with log-normal shadowed. The path-loss law is characterized by a dual pathloss model, assuming the first slope to be $a$ (approximately 2), the second slope to be $d_{s}$ (approximately 4), and the turning point $g$ (between 100 and $200 \mathrm{~m}$ ). The second slope $d_{s}$ $(=a+b)$ is because of an additional attenuation rate coefficient $b$ (approximately 2 ) for the distances greater than $g$. The spectrum efficiency is also evaluated for two different channel situations for the purpose of comparison, namely: i) desired signal as Rician faded and interfering signals as Rayleigh distributed, and ii) both desired and interfering signals as Rician distributed. For a cellular land mobile radio system, spectrum efficiency $\left(E_{s}\right)$ is defined as the ratio of carried traffic per channel $\left(a_{c}\right)$ and the product of bandwidth $(W)$, number of cells per cluster $(C)$, and cells area $S_{a}$ $\left(E_{s} \triangleq a_{c} / W C S_{a}\right.$, erlang $/ \mathrm{MHz} / \mathrm{km}^{2}$ ).

The paper is organized as follows. Section II describes a propagation model for a microcellular system. Section III formulates the co-channel interference probability for microcells for four different propagation characteristics and presents the computational results. In Section IV, spectrum efficiency is evaluated, and, finally, Section $\mathrm{V}$ discusses the conclusions.

\section{PRopagation MODEL}

In the microcellular radio environment, the desired signal level $r$ around the local mean is given by a Rician distribution 
where the probability density function (pdf) is given by

$$
\begin{aligned}
f_{r}\left(r \mid p_{o d}\right) & =\frac{r}{p_{o d}^{\prime}} \exp \left[-\frac{r^{2}+s^{2}}{2 p_{o d}^{\prime}}\right] I_{o}\left(\frac{r s}{p_{o d}^{\prime}}\right) \\
R_{d} & =\frac{s^{2}}{2 p_{o d}^{\prime}} ; \quad p_{o d}=\left(R_{d}+1\right) p_{o d}^{\prime} \\
0 & \leq r<\infty ; \quad s \geq 0
\end{aligned}
$$

where $I_{o}()$ is the modified Bessel function of the first kind and zeroth order, $s$ is the peak value of the specular radio signal, $p_{o d}^{\prime}$ is the average power of the scattered signal, $R_{d}$ is the Rician factor which depends on the ratio of the signal power from the dominant signal path relative to that of the scattered signal, and $p_{o d}$ is the local mean power.

The pdf for the instantaneous signal power $p_{d}$ is given by using $p_{d}=(1 / 2) r^{2}$

$$
f_{p_{d}}\left(p_{d} \mid p_{o d}\right)=\frac{1}{p_{o d}^{\prime}} \exp \left(-\frac{2 p_{d}+s^{2}}{2 p_{o d}^{\prime}}\right) I_{o}\left(\frac{\sqrt{2 p_{d}} s}{p_{o d}^{\prime}}\right) .
$$

Here

$$
p_{o d}^{\prime}=p_{o d} /\left(R_{d}+1\right) .
$$

The slow-varying local mean $p_{o d}$ is given by a log-normal pdf:

$$
f_{p_{o d}}\left(p_{o d}\right)=\frac{1}{\sqrt{2 \pi} \sigma_{d} p_{o d}} \exp \left\{-\left(\ln p_{o d}-m_{d}\right)^{2} / 2 \sigma_{d}^{2}\right\} .
$$

Here, $\sigma_{d}^{2}$ and $\xi_{d}=\exp \left(m_{d}\right)$ are the logarithmic variance and the area mean power of the desired signal, respectively.

The condition pdf for the instantaneous power of a cochannel interference $p_{i}$ is

$$
f_{p_{i}}\left(p_{i} \mid p_{o i}\right)=\frac{1}{p_{o i}} \exp \left(-\frac{p_{i}}{p_{o i}}\right)
$$

where $p_{o i}$ is the local mean of the interfering signal. Equation (4) is obtained because the co-channel interferer is a Rayleigh faded signal.

The pdf for the $p_{o i}$ is described by a density function of the form (3) with the following substitution:

$$
\begin{aligned}
p_{o d} & \rightarrow p_{o i} \\
\sigma_{d} & \rightarrow \sigma_{i} \\
m_{d} & \rightarrow m_{i} \\
\xi_{d} & \rightarrow \xi_{i} .
\end{aligned}
$$

Using a dual path-loss model, the received signal power is given by [12]

$$
p_{r}=C p_{t}\left[\frac{1}{d^{a}\left(1+\frac{d}{g}\right)^{b}}\right]
$$

where $C$ is a constant, $p_{r}$ is the average received power, $p_{t}$ is the average transmitter power, $d$ is the distance between the base station and mobile unit, $g$ is the turning point of the path-loss curve, $a$ is the basic attenuation rate for short distances (approximately 2 ), and $b$ is the additional attenuation rate coefficient (approximately 2) for distances greater than 100 to $200 \mathrm{~m}$.

\section{Co-Channel Interference Probability}

It is imperative to determine the co-channel interference probability for evaluating the spectrum efficiency. Co-channel interference probability is defined as

$$
F(C I) \triangleq \sum_{n} F(C I \mid n) F_{n}(n)
$$

where $F_{n}(n)$ is the probability of $n$ co-channel interferers being active, and $F(C I \mid n)$ is the corresponding conditional co-channel interference probability

$$
F(C I \mid n) \triangleq \operatorname{Prob}\left\{p_{d} / p_{n}<\alpha\right\}
$$

where $p_{n}$ is the composite interference power from $n$ active channels, and $\alpha$ is the specified protection ratio.

The formulation of the conditional co-channel interference probability for four different propagation conditions is described below.

\section{A. Rician Plus Log-Normal Desired Signal and Rayleigh Plus Log-Normal Interfering Signals}

First, the co-channel interference probability is derived for a very general case, which is represented by a Rician plus lognormal desired signal and Rayleight plus log-normal interfering signals in a microcellular environment. This investigation is carried out based on the propagation results reported in [10], which states that in general, the signal fading in microcells can be decomposed into a path-loss component, together with a slow varying component having a log-normal distribution and a fast varying component with a Rician distribution. Accordingly, a three-stage propagation model should be appropriate to describe a microcellular environment: i) area mean depending on the path-loss characteristics in the range from the transmitter to the area where the receiver is located; ii) local mean within that area, which is slow varying, can be very satisfactory represented by a log-normal distribution; and iii) superimposed fast fading instantaneous power which follows a Rician distribution for the desired signal and a Rayleigh distribution for the interfering signal. The coexistence of Rician and log-normal conditions has also been found in the land-mobile satellite channel [21], [22].

The composite pdf for instantaneous power of the desired signal is given by

$$
\begin{aligned}
f_{p_{d}}\left(p_{d}\right)= & \int_{0}^{\infty} \frac{\left(R_{d}+1\right)}{\sqrt{2 \pi} \sigma_{d} p_{o d}^{2}} \\
& \cdot \exp \left[-\frac{\left(R_{d}+1\right)\left(2 p_{d}+s^{2}\right)}{2 p_{o d}}-\frac{\left(\ln p_{o d}-m_{d}\right)^{2}}{2 \sigma_{d}^{2}}\right] \\
& \cdot I_{o}\left(\frac{\sqrt{2 p_{d}} s\left(R_{d}+1\right)}{p_{o d}}\right) d p_{o d}
\end{aligned}
$$

It is worth mentioning here that $s$ is also a function of $p_{o d}$ and is given by $\left[2 R_{d} p_{o d} /\left(R_{d}+1\right)\right]^{1 / 2}$. In the present analysis $s$, the line-of-sight component is considered to be a function of distance $d$, and its variability is included in the system performance calculation by using Rician factor $R_{d}$. It should be noted that the Rician factor varies significantly as the 
distance between the base station and mobile terminal changes [10]. Since in the following analysis interference probability is evaluated for the worst-case situation, computational results are shown only for typical values of Rician factors.

Developing a statistical model of the Rician factor and using it to make detailed study of a microcellular system would be an interesting subject for future study.

The composite pdf for $n$ interfering signals with combined Rayleigh fading and log-normal shadowing [3] is given by using (4) and (5):

$$
\begin{aligned}
f_{p_{n}}\left(p_{n}\right)= & \frac{1}{\sqrt{2 \pi}} \sigma_{n} \int_{0}^{\infty} \frac{1}{p_{o n}^{2}} \\
& \cdot \exp \left[-\frac{\left(\ln p_{o n}-m_{n}\right)^{2}}{2 \sigma_{n}^{2}}-\frac{p_{n}}{p_{o n}}\right] d p_{o n}
\end{aligned}
$$

where $p_{o n}=\sum_{i=1}^{n} p_{o i}, \sigma_{n}^{2}$ and $\xi_{n}=\exp \left(m_{n}\right)$ are the logarithmic and area mean of a log-normal variable, which is approximately equivalent to the sum of $n$ independent $\log$ normal interferers. The parameters $m_{n}$ and $\sigma_{n}^{2}$ are determined using Schwartz and Yeh's method [5], [18].

Using (9) and (10), conditional interference probability is given by

$$
\begin{aligned}
F(C I \mid n)= & \int_{0}^{\infty} \quad d p_{o n} \int_{0}^{\infty} \frac{W}{2 \pi \sigma_{d} \sigma_{n} p_{o d} p_{o n}} \\
\cdot \exp \left[-\frac{\left(\ln p_{o d}-m_{d}\right)^{2}}{2 \sigma_{d}^{2}}-\frac{\left(\ln p_{o n}-m_{n}\right)^{2}}{2 \sigma_{n}^{2}}\right. & \left.-\frac{R_{d} W p_{o d}}{\alpha p_{o n}\left(R_{d+1}\right)}\right] d p_{o d}
\end{aligned}
$$

where

$$
W=\frac{\alpha}{\frac{p_{o d}}{p_{o n}\left(R_{d}+1\right)}+\alpha} .
$$

In (11) $m_{d}=\ln \xi_{d}$ can be obtained using the following relation for the worst case of desired signal to interference ratio:

$$
\xi_{d}=\xi_{i} \frac{R_{u}^{a}\left(G+R_{u}\right)^{b}}{(G+1)^{b}} .
$$

Here $R_{u}$ is the (normalized) reuse distance, which is defined as the ratio of the distance $D$ between the centers of the nearest neighboring co-channel cells and the cell radius $R$ :

$$
R_{u} \triangleq \frac{D}{R}
$$

and $G=g / R$.

In deriving (13) it is assumed that the transmit power $p_{t}$ is the same for desired and interfering mobiles. Equation (13) has two limiting cases.

1) At distances significantly less than the turning point, i.e., $G>R_{u}$, (13) becomes

$$
\frac{\xi_{d}}{\xi_{i}}=R_{u}^{a}
$$

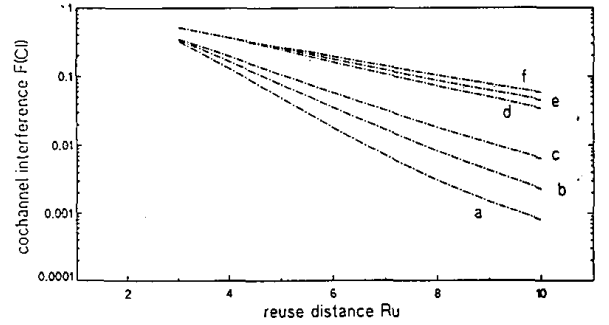

Fig. 1. Co-channel interference probability versus normalized reuse distance for shadowed Rician desired signal and shadowed Rayleigh interfering signals with $A_{c}=5$ erlang, $n_{c}=10, G=0.67, \alpha=8 \mathrm{~dB}, R_{d}=7 \mathrm{~dB}, B=0.02$, $n=6:$ (a) $\sigma_{d}=0 \mathrm{~dB}, \sigma_{i}=6 \mathrm{~dB} ;$ (b) $\sigma_{d}=4 \mathrm{~dB}, \sigma_{i}=6 \mathrm{~dB}$; (c) $\sigma_{d}=6 \mathrm{~dB}, \sigma_{i}=6 \mathrm{~dB}$; (d) $\sigma_{d}=0 \mathrm{~dB}, \sigma_{i}=12 \mathrm{~dB}$; (e) $\sigma_{d}=4 \mathrm{~dB}$ $\sigma_{i}=12 \mathrm{~dB}$; (f) $\sigma_{d}=6 \mathrm{~dB}, \sigma_{i}=12 \mathrm{~dB}$.

2) At distances greater than the turning point, i.e., $G<1$, (13) reduces to

$$
\frac{\xi_{d}}{\xi_{i}}=R_{u}^{a+b}
$$

Equations (13)-(16) are based on the propagation model reported in [12], and, accordingly, the turning point $g$ lies between 100 and $200 \mathrm{~m}$.

Co-channel interference probability can be computed using (7), (11), (13), and $F_{n}(n)$. It is assumed that all co-channel interferers are statistically independent and identically distributed. Further, it is assumed that only interfering signals from the nearest neighboring six co-channel cells are considered, the blocking probability $B$ is the same in all cells, and the channel is uniformly loaded. Accordingly, $F_{n}(n)$ is given as

$$
F_{n}(n)=\left(\begin{array}{l}
6 \\
n
\end{array}\right) a_{c}^{n}\left(1-a_{c}\right)^{6-n}
$$

where $a_{c}=A_{c} / n_{c}, n_{c}$ is the number of channels per cell, and $A_{c}$ is the carried traffic per cell defined as

$$
A_{c} \triangleq A(1-B)
$$

Here $A$ is the offered traffic per cell in erlang, and $B$ is the blocking probability which is determined using Erlang $B$ formula [19], [20]:

$$
B=\frac{A^{n_{c}}}{n_{c} ! \sum_{n=0}^{n_{c}}\left(\frac{A^{n}}{n !}\right)} .
$$

Using (7)-(19), co-channel interference probability is calculated for six interferers and shown in Figs. 1-3. Fig. 1 shows co-channel interference probability $F(C I)$ versus reuse distance $R_{u}$ for $A_{c}=5$ erlang, $n_{c}=10, G=0.67, \alpha=8 \mathrm{~dB}$, and $R_{d}=7 \mathrm{~dB}$ with the spread of desired and interfering signals as parameters. It can be seen from Fig. 1 that, in the interfering signal with a spread of $6 \mathrm{~dB}, F(C I)$ increases with 


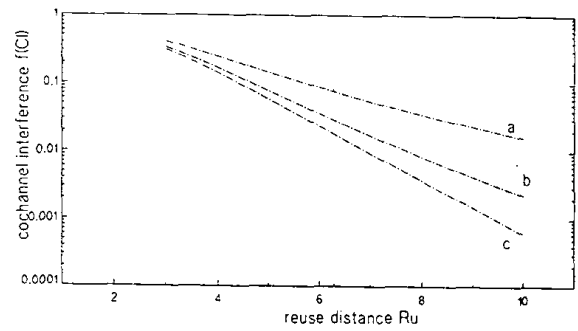

Fig. 2. Co-channel interference probability versus normalized reuse distance for shadowed Rician desired signal and shadowed Rayleigh interfering signals with $A_{c}=5$ erlang, $n_{c}=10, G=0.67, \alpha=8 \mathrm{~dB}, B=0.02, \sigma_{d}=4 \mathrm{~dB}$, $\sigma_{i}=6 \mathrm{~dB}, n=6$ : (a) $R_{d}=0$; (b) $R_{d}=7 \mathrm{~dB}$; (c) $R_{d}=12 \mathrm{~dB}$.

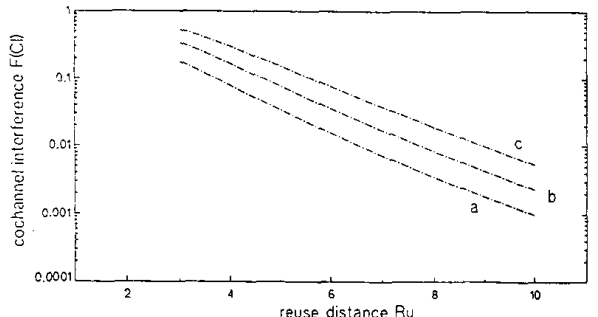

Fig. 3. Co-channel interference probability versus normalized reuse distance for shadowed Rician desired signal and shadowed Rayleigh interfering signals with $n_{c}=10, G=0.67, \alpha=8 \mathrm{~dB}, \sigma_{d}=4 \mathrm{~dB}, \sigma_{i}=6 \mathrm{~dB}, R_{d}=7 \mathrm{~dB}$, $G=0.67, n=6$ : (a) $A_{c}=2.3$ erlang; (b) $A_{c}=5$ erlang; (c) $A_{c}=9.9$ erlang.

the increase of spread of desired signal by a higher value than in the case where the interfering signal has a spread of $12 \mathrm{~dB}$.

Co-channel interference probability versus distance as shown in Fig. 2 for Rician factor $R_{d}$ as a parameter, $G=0.67$, $\alpha=8 \mathrm{~dB}, A_{c}=5$ erlang, $\sigma_{d}=4 \mathrm{~dB}, \sigma_{i}=6 \mathrm{~dB}$, and $n_{c}=10 . R_{d}=0$ represents the conditions for a macrocellular environment. The computational results for Fig. 2 are obtained using (7), (11), and (17)-(19) and six interferers. It is seen from Fig. 2 that for a given value of reuse distance, $F(C I)$ is lower for a microcellular system than for a macrocellular system, and as $R_{u}$ increases $F(C I)$ decreases. Therefore, to maintain a specific value of $F(C I)$, a much smaller value of the reuse distance is adequate for a microcellular system as compared with the conventional macrocellular system.

Fig. 3, which is obtained for six interferers using (7), (11), and (17)-(19), depicts the performance of a microcellular system in terms of co-channel interference probability $F(C I)$ for $\alpha=8 \mathrm{~dB}, R_{d}=7 \mathrm{~dB}, n_{c}=10, \sigma_{d}=4 \mathrm{~dB}, \sigma_{i}=6 \mathrm{~dB}$, $G=0.67$, and carried traffic as a parameter. It is seen from Fig. 3 that a higher value of $A_{c}$ increases $F(C I)$ at a given reuse distance.

\section{B. Rician Desired Signal and Rayleigh Plus Log-Normal Interfering Signals}

Conditional co-channel interference probability for a Rician desired signal and Rayleigh plus log-normal interfering signals can be obtained from (11) after removing the shadowed condition on the local mean power $p_{\text {od }}$ of the desired signal,

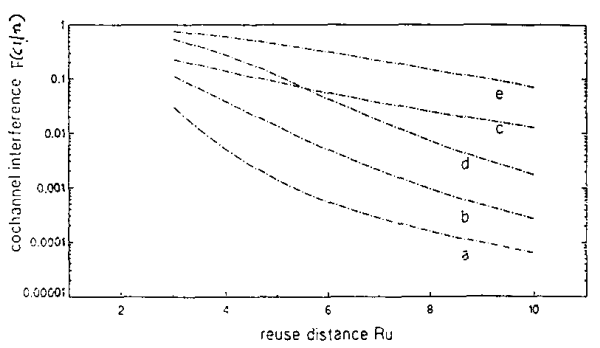

Fig. 4. Co-channel interference probability versus normalized reuse distance for Rician faded desired signal and Rayleigh plus log-normal shadowed interfering signals with $\alpha=8 \mathrm{~dB}, A_{c}=5$ erlang, $n_{c}=10, B=0.02$, $G=0.67, R_{d}=7 \mathrm{~dB}$ : (a) $n=1$ interfering signal, spread $\sigma=0 \mathrm{~dB}$; (b) $n=1, \sigma=6 \mathrm{~dB}$; (c) $n=1, \sigma=12 \mathrm{~dB}$; (d) $n=6, \sigma=6 \mathrm{~dB}$; (e) $n=6, \sigma=12 \mathrm{~dB}$.

i.e., $p_{o d}$ is a constant.

$$
\begin{aligned}
F(C I \mid n)= & \int_{0}^{\infty} \frac{W}{\sqrt{2 \pi} \sigma_{n} p_{o n}} \\
& \cdot \exp \left[-\frac{\left(\ln p_{o n}-m_{n}\right)^{2}}{2 \sigma_{n}^{2}}-\frac{R_{d} W p_{o d}}{\alpha p_{o n}\left(R_{d}+1\right)}\right] d p_{o n}
\end{aligned}
$$

Using (7), (17)-(19), and (20), co-channel interference probability is evaluated and shown in Fig. 4.

Fig. 4 shows the effect of shadowing spread $\sigma$ on co-channel interference probability for $\alpha=8 \mathrm{~dB}, R_{d}=7 \mathrm{~dB}$, and $G=0.67$ with $n=1$ and $n=6$ active co-channel interferers. As expected, higher values of spread cause higher interference probability at a particular reuse distance for both $n=1$ and $n=6$. Fig. 4 also confirms that $n=1$ yields lower value of $F(C I)$ for a given value of reuse distance than co-channel interference due to $n=6$ interferers.

\section{Rician Desired Signal and Rayleigh Interfering Signals}

In the case of a Rician desired signal and Rayleigh interfering signals, the conditional co-channel interference probability can be obtained from (11). However, instead of using (11), a simple, direct, and exact method for the evaluation of $F(C I \mid n)$ for this case is given below.

In the case of $n$ Rayleigh independent interfering signals, the pdf for the joint interference power $p_{n}$ is obtained by convolving (4) $n$ times and given by a gamma distribution:

$$
f_{p_{n}}\left(p_{n}\right)=\frac{1}{p_{o i}} \frac{\left(p_{n} / p_{o i}\right)^{n-1}}{(n-1) !} \exp \left(-\frac{p_{n}}{p_{o i}}\right) \text {. }
$$

Equation (21) is obtained by assuming that each interferer has equal local mean power $p_{o i}$ and $p_{n}=\sum_{i=1}^{n} p_{i}$.

Using (4), (8), and (21), the conditional interference probability is given by

$$
\begin{aligned}
F(C I \mid n)= & W \exp \left[-R_{d} W \frac{p_{o d}^{\prime}}{\alpha p_{o i}}\right] \\
& \cdot \sum_{k=0}^{n-1}\left(W \frac{p_{o d}^{\prime}}{\alpha p_{o i}}\right)^{k} L_{k}(-k W)
\end{aligned}
$$




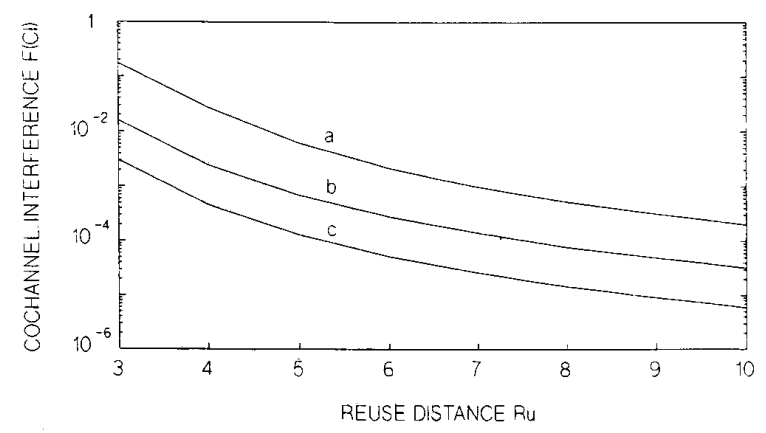

Fig. 5. Co-channel interference probability versus normalized reuse distance for Rician faded desired sighlal with protection ratio $\alpha=8 \mathrm{~dB}$, blocking probability, $B=0.02$, carried traffic per cell, $A_{c}=5$ erlang, $n_{c}=10$ channels per cell, Rician factor $R_{d}=7 \mathrm{~dB}$, and $G=g / R=0.67$ : (a) six Rayleigh co-channel interferers; (b) one Rayleigh co-channel interferer; and (c) one Rician co-channel interferer.

where $L_{k}=$ Laguerre polynomial [16], [17] and $W$ can be obtained from (12) after substituting $p_{o i}$ for $p_{o n}$.

Co-channel interference probability has been computed using (7), (17)-(19), and (22) for $\alpha=8 \mathrm{~dB}, A_{c}=5$ erlang, $R_{d}=7 \mathrm{~dB}, G=0.67$ and $n_{c}=10$ for $n=1,6$ as a function of $R_{u}$ and is shown in Fig. 5. For $n=1$, (22) reduces to

$$
F(C I \mid 1)=W \exp \left[-R_{d} W \frac{p_{o d}^{\prime}}{\alpha p_{o i}}\right]
$$

For the purpose of comparison, computational results for one co-channel interferer with Rician pdf are also included. $F(C I \mid n)$ for a single Rician co-channel interferer is given by

$$
\begin{aligned}
F_{r}(C I \mid 1)= & \frac{W}{\alpha}\left\{1-Q\left(\left[\frac{2 R_{d} W}{\alpha}\right]^{\frac{1}{2}},\left[2 R_{d} W\right]^{\frac{1}{2}}\right)\right\} \\
& +W Q\left(\left[2 R_{d} W\right]^{\frac{1}{2}},\left[\frac{2 R_{d} W}{\alpha}\right]^{\frac{1}{2}}\right)
\end{aligned}
$$

Here $Q(a, b)$ is a Marcum's Q-function.

It is seen from Fig. 5 that co-channel interference probability is always minimum for one Rician interferer.

\section{Desired and Interfering Signals as Rician pdf}

Finally, the conditional co-channel interference probability is derived for a special case in which desired and interfering signals are Rician pdf If the pdf for the interfering signals is also Rician, the pdf for the instantaneous power is of the same form as (2) with the simple substitutions of changing index $d$ to $i$.

In a microcellular system, generally interfering signals are Rayleigh faded because they travel a longer distance to arrive at the base station. But in the case of a very small microcell size, the distance between the centers of co-channel cells may not be large, and, therefore, the interfering signals may be modeled by Rician fading. For example, if $R=200 \mathrm{~m}$, and

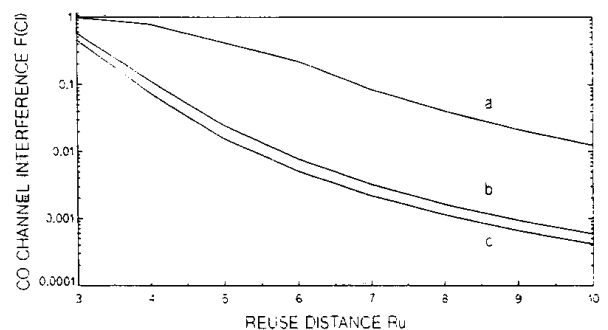

Fig. 6. Co-channel interference probability versus normalized reuse distance for Rician faded desired and interfering signals with $A_{c}=5$ erlang, $n_{c}=10$, $\alpha=8 \mathrm{~dB}, R_{d}=7 \mathrm{~dB}, n=6$; (a) $G=10$; (b) $G=1$; (c) $G=0.67$.

$R_{u}=3$, then $D=600 \mathrm{~m}$. For such cases, assuming the pdf for interfering signals as Rician distributed can be justified.

Assuming $n$-Rician independent interfering signals, the pdf for the joint interference $p_{n}$ is given by

$$
\begin{aligned}
f_{p_{n}}\left(p_{n}\right)= & \frac{1}{p_{o i}^{\prime}}\left(\frac{2 p_{n}}{p}\right)^{\frac{n-1}{2}} \\
& \cdot \exp \left(-\frac{2 p_{n}+p}{2 p_{o i}^{\prime}}\right) I_{n-1}\left(\frac{\sqrt{2 p_{n} p}}{p_{o i}^{\prime}}\right)
\end{aligned}
$$

where $p=\sum_{i=1}^{n} s_{i}^{2}$ and $p_{n}=\sum_{i=1}^{n} p_{i}$

The conditional co-channel interferehce probability is obtained using (2), (8), and (25), assuming $R_{d}=R_{i}$ :

$$
\begin{aligned}
F(C I \mid n)= & 1-\left(\frac{1}{\sqrt{2 n R_{d}}}\right)^{n-1} \int_{0}^{\infty} u^{n} \\
& \cdot \exp \left(-\frac{u^{2}+2 n R_{d}}{2}\right) I_{n-1}\left(u \sqrt{2 n R_{d}}\right) \\
& \cdot Q\left(\sqrt{2 R_{d}}, u\left(\alpha p_{o i} / p_{o d}\right)^{\frac{1}{2}}\right) d u
\end{aligned}
$$

where $Q(x, y)$ is a Marcum's Q-function.

Computational results are obtained for $F(C I)$ versus $R_{u}$ for $R_{d}=7 \mathrm{~dB}$ and $12 \mathrm{~dB}, \alpha=8 \mathrm{~dB}$, and $G$ as a parameter. Fig. 6 reconfirms for this case also that i) the higher the reuse distance, the less the co-channel interference probability; ii) $F(C I)$ is dependent on $G$; and iii) $F(C I)$ decreases with increase in $R_{d}$. (Results are not shown for $R_{d}=12 \mathrm{~dB}$ in Fig. 6.)

\section{SPECTRUM EFFICIENCY}

The service area of a cellular radio system is divided into a regular array of hexagonal cells. These cells are grouped in identical clusters, where each cluster occupies the total bandwidth available for the mobile radio system. With the number of channels per cell $n_{c}$, carried traffic per cell $A_{c}$, bandwidth per channel $W$, and cluster size $C$ cells, given a unit cell are of $S_{a}$, spectrum efficiency $E_{s}$ is defined as

$$
E_{s} \triangleq \frac{A_{c}}{n_{c} W C S_{a}} \operatorname{erlang} / \mathrm{MHz} / \mathrm{km}^{2}
$$




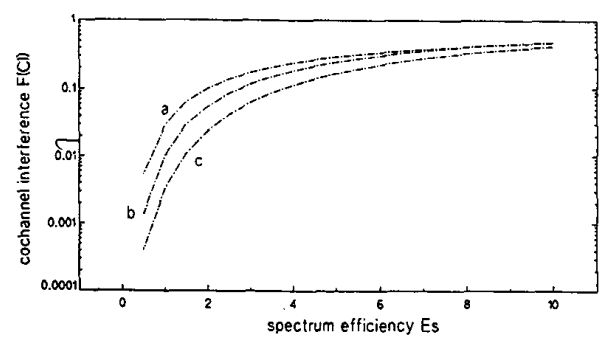

Fig. 7. Co-channel interference probability versus spectrum efficiency for shadowed Rician desired signal and shadowed Rayleigh interfering signals with $\sigma_{d}=4 \mathrm{~dB}, \sigma_{i}=6 \mathrm{~dB}, R_{d}=7 \mathrm{~dB}, \alpha=8 \mathrm{~dB}, n_{c}=10$ $W=25 \mathrm{KHz}, S_{a}=1 \mathrm{~km}^{2}, G=0.67, n=6$ : (a) $A_{c}=2.3$ erlang; (b) $A_{c}=5$ erlang; (c) $A_{c}=9.9$ erlang.

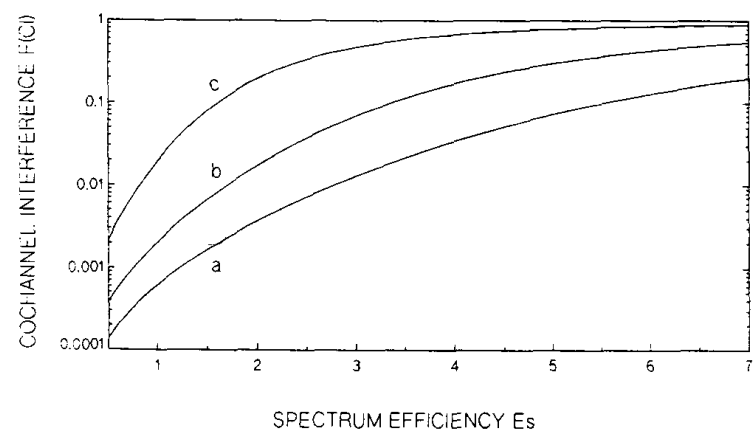

Fig. 8. Co-channel interference probability versus spectrum efficiency for Rician desired signal and Rayleigh interfering signals with $R_{d}=7 \mathrm{~dB}$ $n_{c}=10, A_{c}=5$ erlang, $G=0.67, B=0.02, S_{a}=1 \mathrm{~km}^{2}, n=6$, $W=25 \mathrm{KHz}$ : (a) $\alpha=8 \mathrm{~dB}$; (b) $\alpha=12 \mathrm{~dB}$; (c) $\alpha=18 \mathrm{~dB}$.

For a hexagonal system, the reuse distance and the number of cells per cluster are related by [1]

$$
R_{u}=(3 C)^{\frac{1}{2}} .
$$

The cells are assumed to form a cluster of size $C$, located around the reference cell and repeated around each of its co-channel cells. The cluster size is taken on the form:

$$
C=i^{2}+i j+j^{2} ; \quad i, j \geq 0
$$

with integers $i$ and $j$.

Equations (28) and (29) convey to us that the spectrum efficiency is inversely proportional to the square of the reuse distance. Therefore, it can be concluded from Fig. 2 that for a given value of $F(C I)$, spectrum efficiency is higher for a microcellular system than that for a macrocell, and $E_{s}$ in a microcell increases with Rician factor $R_{d}$.

The effect of carried traffic on spectrum efficiency is shown in Fig. 7 for a microcellular radio system having shadowed Rician desired signal and shadowed Rayleigh interfering signals. The results are similar to the macrocellular system, i.e., the spectrum efficiency increases with the carried traffic, given a fixed interference probability (practical value $\leq 0.1$ ). Computational results were also obtained for three other

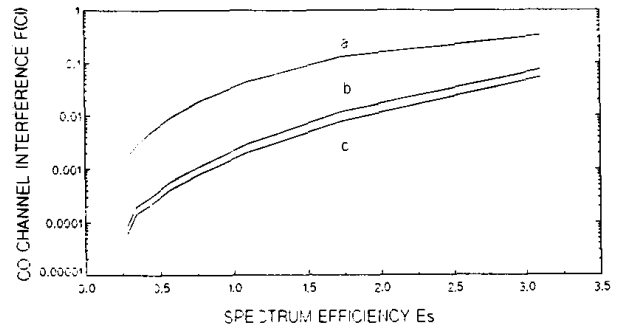

Fig. 9. Co-channel interference probability versus spectrum efficiency for Rician desired signal and interfering signals with $\alpha=8 \mathrm{~dB}, A_{c}=2.3$ erlang, $n_{c}=10, W=25 \mathrm{KHz}, S_{a}=1 \mathrm{~km}^{2}, R_{d}=R_{i}=7 \mathrm{~dB}, n=6:$ (a) $G=10$; (b) $G=1$; (c) $G=0.67$.

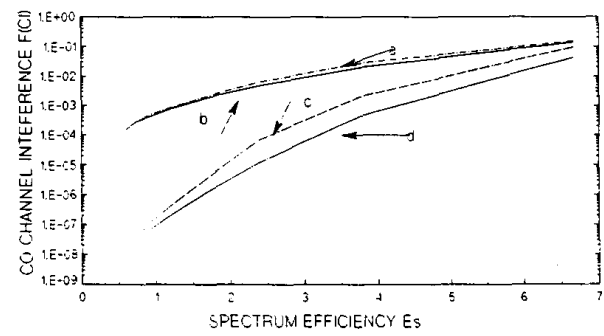

Fig. 10. Comparison of co-channel interference probability versus spectrum efficiency in case of Rician fading interferers with Rayleigh fading interferers for $\alpha=8 \mathrm{~dB}, A_{c}=5$ erlang, $n_{c}=10, n=6, W=25 \mathrm{KHz}$, $S_{a}=1 \mathrm{~km}^{2}, G=0.67$ : (a) $R_{d}=7 \mathrm{~dB}$, Rayleigh interference $\left(R_{i}=0\right)$; (b) $R_{d}=R_{i}=7 \mathrm{~dB}$; (c) $R_{d}=12 \mathrm{~dB}, R_{i}=0$; (d) $R_{d}=R_{i}=12 \mathrm{~dB}$.

microcellular propagation conditions, and similar conclusions were drawn.

Fig. 8 depicts the effect of a protection ratio on the spectrum efficiency for $R_{d}=7 \mathrm{~dB}, A_{c}=5$ erlang, $n_{c}=10$, $W=25 \mathrm{KHz}$, and $S_{a}=1 \mathrm{~km}^{2}$, considering Rician desired signal and Rayleigh interfering signals, Clearly, the spectrum efficiency can be increased by tolerating a lower value of the protection ratio.

The influence of $G=g / R$ on the spectrum efficiency can be seen in Fig. 9 for $R_{d}=7 \mathrm{~dB}, \alpha=8 \mathrm{~dB}, A_{c}=5$ erlang, $n_{c}=10, W=25 \mathrm{KHz}$, and $S_{a}=1 \mathrm{~km}^{2}$, considering both desired and interfering signals as Rician faded. For a particular value of $F(C I)$, spectrum efficiency increases with decreasing $G$. For designing an efficient microcellular system with a certain value of $G$, a compromise has to be made between spectrum efficiency and an acceptable value of interference probability.

Fig. 10 compares the co-channel interference probability versus spectrum efficiency in the case of Rician fading interferers with Rayleigh fading interferers for a $\alpha=8 \mathrm{~dB}, A_{c}=$ 5 erlang, $n_{c}=10, W=25 \mathrm{KHz}, S_{a}=1 \mathrm{~km}^{2}, G=0.67$, and $R_{d}$ as a parameter. It can be seen from Fig. 10 that Rician fading interferers result in a better spectrum efficiency than Rayleigh fading interferers. Comparison between spectrum efficiency due to Rician fading interferers and Rayleigh fading interferers for $G=10$ and $G=1$ is presented in Fig. 11. It can be seen from Fig. 11 that now spectrum efficiency is higher for Rayleigh fading interferers than for Rician fading interferers. 


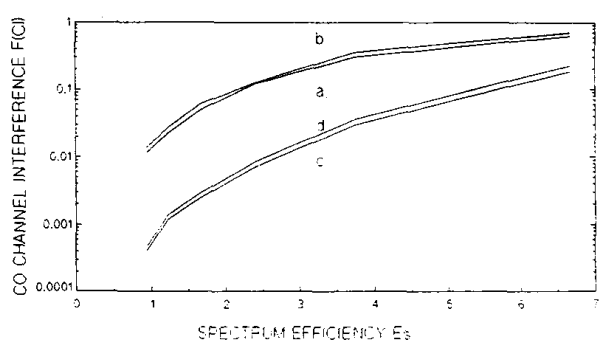

Fig. 11. Comparison of co-channel interference probability versus spectrum efficiency in case of Rician fading interferers with Rayleigh fading interference for $\alpha=8 \mathrm{~dB}, A_{c}=5$ erlang, $n_{c}=10, n=6, W=25 \mathrm{KHz}, S_{a}=1 \mathrm{~km}^{2}$, $R_{d}=7 \mathrm{~dB}$ : (a) $G=10, R_{i}=0$; (b) $G=10, R_{i}=7 \mathrm{~dB}$; (c) $G=1$ $R_{i}=0$; (d) $G=1, R_{i}=7 \mathrm{~dB}$.

Comparing Figs. 10 and 11, it can be concluded that Rician fading interferers cause higher efficiency for $G<1$ and less efficiency for $G \geq 1$.

\section{CONCLUSIONS}

A mathematical model is developed to evaluate the cochannel interference probability in a microcellular radio system having Rician faded and log-normal shadowed desired signal and Rayleigh faded and log-normal shadowed interfering signals by considering a dual path-loss model with a turning point. The co-channel interference probability thus obtained is used to study the effects of carried traffic, protection ratio, shadowing spread, Rician factor, and turning point $(G=g / R)$ on spectrum efficiency. The value of the turning point varies between 100 and $200 \mathrm{~m}$. As a special case, performance is also evaluated for Rician faded desired and interfering signals.

Computational results show that slow fading (shadowing) causes increase in the co-channel interference probability as compared with the case in which interfering signals are Rayleigh faded only. Further, by introducing shadowing in both the desired and interfering signals, it is found that, with the same value of spread for the desired signal and a different spread of the interfering signal, the increase in the co-channel interference probability is less profound with a higher spread of interfering shadowing.

In the case of Rician faded interferers, performance of a microcellular system improves as compared with the systems having Rayleigh faded interferers; for $G<1$ and for $G \geq 1$ the situation gets reversed; i.e., Rayleigh faded interferers yield superior performance.

The spectrum efficiency of a microcell for practical values of the interference probability $(\leq 0.1)$ increases with the increase in carried traffic, i.e., blocking probability. As expected, the microcells offer higher spectrum efficiency than that for macrocells for a specific value of co-channel interference probability. Here all the computational results are presented for protection ratio $\alpha=8 \mathrm{~dB}$ (except for Fig. 8) because this value is suitable for digital modulated systems [3]. For analog FM systems, $\alpha$ is generally about $18 \mathrm{~dB}$ [3]. Results are shown in Fig. 8.

The present study would be useful for designers and planners in developing any type of microcellular system (e.g., streets, highways, forests, rural areas etc.). Since Rician interfering signals are generally present in picocells, the analytical model developed in this paper can also be used to study indoor communications.

\section{ACKNOWLEDGMENT}

The authors are grateful to J. C. Olsthoorn, O. L. van Linden, and M. B. Loog for the computational results presented in this paper, and to Mrs. Eefje Ooms for preparing the typescript. They would like to thank the anonymous reviewers for their valuable suggestions.

\section{REFERENCES}

[1] V.H. MacDonald, "The cellular concept," Bell Syst. Tech. J., vol. 58, pp. 15-41, Jan. 1979.

[2] W.C.Y. Lee, Mobile Cellular Telecommunications Systems. New York: McGraw-Hill, 1989.

[3] R. Prasad and A. Kegel, "Improved assessment of interference limits in cellular radio performance," IEEE Trans. Veh. Technol., vol. 40, pp. 412-419, May 1991

[4] R. Prasad, A. Kegel and J.C. Arnbak, "Analysis of system performance of high-capacity mobile radio," in Proc. 39th IEEE Veh. Technol. Conf., San Francisco, CA, pp. 306-310, May 1989.

[5] R. Prasad and J.C. Arnbak, "Comments on analysis for spectrum efficiency in single cell trunked and cellular mobile radio," IEEE Trans. Veh. Technol., vol. 37, pp. 220-222, Nov. 1988.

[6] Y.-D. Yao and A. V. H. Sheikh, "Outage probability analysis for microcell mobile radio systems with co-channel interferers in Rician/Rayleigh fading environment," Electron. Lett., vol. 26, pp. 864-866, June 1990.

[7] R. H. Muammar, "Co-channel interference in microcellular mobile radio system," in Proc. 4Ist IEEE Veh. Technol. Conf., St. Louis, MO, pp. 198-203, May 1991.

[8] R. Prasad and A. Kegel, "Spectrum efficiency of microcellular systems," in Proc. 41st IEEE Veh. Technol. Conf., St. Louis, MO, pp. 357-361, May 1991.

[9] R. Prasad, A. Kegel, and J. C. Olsthoorn, "Spectrum efficiency analysis for microcellular mobile radio systems," Electron. Lett., vol. 27 , pp. $423-424$, Feb. 1991.

[10] S.T.S. Chia, "Radiowave propagation and handover criteria for microcells," Br. Telecom. Technol. J., vol. 8, pp. 50-61, Oct. 1990.

[11] E. Green, "Radio link design for microcellular systems," Br. Telecom. Technol. J., vol. 8, pp. 85-96, Jan. 1990.

[12] H. Harley, "Short distance attenuation measurements at $900 \mathrm{MHz}$ and $1.8 \mathrm{GHz}$ using low antenna heights for microcells," IEEE J. Select. Areas Commun., vol. 7, pp. 5-10, Jan. 1989.

[13] R. J.C. Bultitude and G. K. Bedal, "Propagation characteristics on microcellular urban mobile radio channels at $910 \mathrm{MHz}$," IEEE J. Select. Areas. Commun., vol. 7, pp. 31-39, Jan. 1989.

[14] S. T.S. Chia, R. Steel, E. Green, and A. Baran, "Propagation and bit error ratio measurements for a microcellular system," J. Inst. Elect. Radio Eng., vol. 57, no. 6 (supplement), pp. S255-266, Nov./Dec. 1987.

[15] R. Davies, A. Simpson, and J.P. McGreechan, "Propagation measurements at $1.7 \mathrm{GHz}$ for microcellular urban communications," Electron. Lett., vol. 26, pp. 1053-1055, July 1990.

[16] M. Abramowitz and I. A. Stegun Eds. Handbook of Mathematical Functions with Formulas, Graphs and Mathematical Tables. New York: Dover, 1972.

[17] H. L. van Trees, Detection, Estimation, and Modulation Theory. New York: Wiley, 1971.

[18] S. C. Schwartz and Y.S. Yeh, "On the distribution function and moments of power sums with log-normal components," Bell Syst. Tech. J., vol. 61, pp. 1441-1402, Sept. 1982.

[19] J.A. Elldin and G. Lind, Elementary Telephone Traffic Theory. Stockholm, Sweden: Telefona ktiebolaget LM ERICSSON, 1967.

[20] M. Schwartz, Telecommunication Networks Protocols, Modeling and Analysis. Reading, MA: Addison-Wesley, 1987.

[21] C. Loo, "Digital transmission through a land-mobile satellite channel," IEEE Trans. Commun., vol. 38, pp. 693-697, May 1990.

[22] D. J. R. van Nee, H. S. Misser, and R. Prasad, "Direct-sequence spread spectrum in a shadowed Rician fading land-mobile satellite channel," IEEE J. Select. Areas Commun., vol. 10, pp. 350-357, Feb. 1992. 


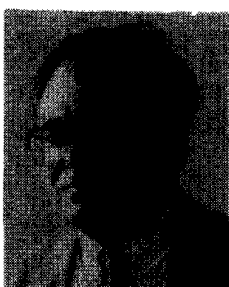

Ramjee Prasad (M'88 - SM'90) was born in India in 1946. He received the B.Sc. (Eng.) degree from Bihar Institute of Technology, Sindri, India, and the M.Sc. (Eng.) and Ph.D. degrees from Birla Institute of Technology (BIT), Ranchi, India, in 1968, 1970, and 1979 , respectively.

$\mathrm{He}$ joined BIT as a Senior Research Fellow in 1970 and became an Associate Professor in 1980. While he was with BIT, he supervised many research projects in the area of microwave and plasma engineering. From 1983 to 1988 he was with the University of Dar es Salaam (UDSM), Tanzania, where he became a Professor in Telecommunications in the Department of Electrical Engineering in 1986. At UDSM he was responsible for the collaborative project "Satellite Communications for Rural Zones" for Eindhoven University of Technology, The Netherlands. Since February 1988, he has been with the Telecommunications and Traffic Control Systems Group, Delft University of Technology The Netherlands, where he is actively involved in the area of mobile and indoor radio communications. His current research interest lies in packe communications, adaptive equalizers, spread-spectrum CDMA systems, and multi-media communications.

Professor Prasad has served as a member of advisory and program committees of several IEEE International conferences. He has also presented tutorials on mobile and indoor radio communications at various universities, technical institutions, and IEEE conferences. He is a member of a working group of European cooperation in the field of scientific and technical research (COST231) on a project dealing with "Evolution of Land Mobile Radio (including personal) Communications" as an expert representing the Netherlands. He is listed in the U.S. Who's Who in the World. He is an Organizer and Interim Chairman of the IEEE Vehicular Technology/Communications Society Join Chapter, Benelux Section. He is an Associate Technical Editor of IEEE Communications Magazine, a Fellow of the Institution of Electronics \& Telecommunication Engineers, and a member of the New York Academy of Sciences. He has published over 100 technical papers.

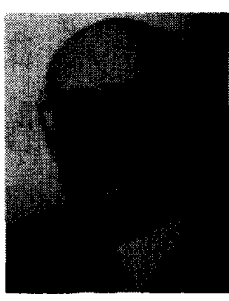

Adriaan Kegel (M'91) was born in The Netherlands in 1932. He received the M.Sc.E.E. degree from Delft University of Technology, The Netherlands, in 1972.

He joined Philips Telecommunications Industry in 1954, where he worked on the development of microwave link equipment as a Radio Design Engineer. In 1964 he joined Delft University of Technology, where he is currently an Associate Professor. In 1970 he became chairman of the

"Working Group Indonesia," which developed an educational broadcasting system called "Teleboard." For this development, the working group received an award from the Scientific Radio Foundation Veder. After completion of the Indonesian Project in 1974, he continued his research work on the coding and transmission of audio-graphic information. This work led to the development of several systems (e.g. Vidiboard) and a number of publications (e.g. on differential chain coding). His current interes is in research and education on mobile communication systems.

Mr. Kegel is a member of NERG (the Netherlands Electronics and Radio Society) and FITCE (the Federation of Telecommunication Engineers of the European Community). 